\title{
EP-145
}

\section{Laparoscopic assisted trans-gastric endoscopic retrograde cholangiopancreatography (LA-ERCP) for postoperative bile leakage: A case report}

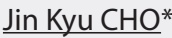

Department of Surgery, Gyeongsang National University Hopstial, Jinju, Korea

Introduction: Laparoscopic assisted trans-gastric endoscopic retrograde cholangiopancreatography (LA-ERCP) is a safe and effective technique for cholelithiasis. We report a successful treatment case through LA-ERCP in patient with bile leakage after laparoscopic common bile duct (CBD) exploration because of the patient's limited degree of mouth opening due to trismus and limb stiffness.

Methods: Here we present a case of a 51-year-old male patient with trismus, limb stiffness after suffering from poliomyelitis. The patient showed acute calculous cholecystitis and acute cholangitis with distal CBD stones. He was unable to receive trans-oral ERCP or percutaneous trans-hepatic biliary drainage (PTBD) because of the limited degree of mouth opening due to trismus and stiffness in his both arm across his chest. So we performed laparoscopic cholecystectomy and removed distal CBD stones through CBD exploration. On the fourth day after the operation, bile leakage was observed. Because he was unable to receive trans-oral ERCP or PTBD, we decided to perform LA-ERCP.

Results: We performed LA-ERCP and successfully inserted endoscopic retrograde bile duct drainage (ERBD) into the common bile duct. During the procedure, the endoscope was introduced through a gastrostomy, obviating the need for an intragastric trocar. The patient underwent ERBD. The patient was discharged on 11 postoperative day without any complications.

Conclusions: For the patients in whom trans-oral and trans-hepatic route are unavailable, LA-ERCP represents an effective approach for the management of biliary complications after laparoscopic cholecystectomy and offers a novel solution. 